\title{
Melatonin promotes the BMP9-induced osteogenic differentiation of mesenchymal stem cells by activating the AMPK/ $\beta$ - catenin signalling pathway
}

Tianyuan Jiang ${ }^{1}$, Chao Xia ${ }^{1}$, Xiaoting Chen ${ }^{1}$, Yan $\mathrm{Hu}^{1}$, Yan Wang ${ }^{1}$, Jin $\mathrm{Wu}^{2}$, Shuyan Chen ${ }^{1 *}$ and Yanhong Gao ${ }^{{ }^{*}}$

\begin{abstract}
Background: Mesenchymal stem cells (MSCs) play a crucial role in maintaining the dynamic balance of bone metabolism. Melatonin may have a regulatory effect on bone metabolism by regulating the lineage commitment and differentiation signalling pathways of MSCs. Among the BMP families, the osteogenesis of BMP9 is considered to be one of the strongest in MSCs. Here, we explored whether melatonin and BMP9 act synergistically on MSC osteogenic differentiation.

Methods: The C3H10T1/2 osteogenic differentiation function induced by melatonin synergizes with BMP9, as detected by the expression of osteogenic markers at different periods. The result was further confirmed by foetal limb explant culture and in vivo stem cell implantation experiments. The effects of the AMPK/ $\beta$-catenin pathway on the osteogenic differentiation of $\mathrm{C} 3 \mathrm{H} 10 \mathrm{~T} 1 / 2$ cells were evaluated by Western blotting.

Results: Melatonin combined with BMP9 significantly enhanced the expression of osteogenic markers at different periods in $\mathrm{C} 3 \mathrm{H} 10 \mathrm{~T} 1 / 2$ cells, effectively enhancing BMP9-induced bone formation in cultured foetal explants and ectopic bone formation in vivo in stem cell transplantation experiments. Melatonin increases the expression of BMP9 in C3H10T1/2 cells and induces Smad1/5/8 translocation from the cytoplasm to the nucleus. In addition, melatonin and BMP9 synergistically promote AMPK and $\beta$-catenin phosphorylation, which can be largely eliminated by AMPK siRNA pretreatment.
\end{abstract}

Conclusions: Melatonin and BMP9 in C3H10T1/2 cells synergistically promote osteogenic differentiation at least in part by activating the AMPK/ $\beta$-catenin signalling pathway.

Keywords: Melatonin, BMP9, Osteogenic differentiation, Mesenchymal stem cells

\section{Background}

Mesenchymal stem cells (MSCs) are pluripotent stem cells derived from mesoderm that can differentiate into osteoblasts, adipocytes and chondrocytes with strong self-renewal and multi-directional differentiation $[1,2]$. Bone is one of the few organs that retains the potential for regeneration and can be continuously remodelled throughout life [3]. The osteogenic differentiation of

\footnotetext{
* Correspondence: chenshuyan@xinhuamed.com.cn;

gaoyanhong@xinhuamed.com.cn; yhgao2010@yahoo.com

${ }^{1}$ Department of Geriatrics, Xinhua Hospital, Shanghai Jiaotong University

School of Medicine, Shanghai 200092, China

Full list of author information is available at the end of the article
}

MSCs plays an important role in bone regeneration and remodelling. This process is regulated by a variety of hormones, transcription factors and cellular signalling pathways, including bone morphogenetic protein (BMP), Wnt, insulin-like growth factor (IGF), epidermal growth factor and growth hormone, and is involved in multiple pathway interactions [3-6].

Bone morphogenetic proteins (BMPs) are important signalling pathways for cell proliferation and differentiation during embryonic development and play a key role in regulating the osteogenic differentiation of mesenchymal stem cells [7]. BMPs belong to the transforming growth factor (TGF) superfamily and have at least 15

(C) The Author(s). 2019 Open Access This article is distributed under the terms of the Creative Commons Attribution 4.0 International License (http://creativecommons.org/licenses/by/4.0/), which permits unrestricted use, distribution, and 
different subtypes present in humans. BMP2, BMP4, BMP7 and BMP9, all play an important role in promoting osteogenic differentiation and bone formation. Among these proteins, BMP2 and BMP7 have been evaluated in clinical trials for the treatment of tibial fractures and spinal fusion $[8,9]$. BMP9 is considered to be one of the most effective factors for inducing mesenchymal stem cell osteogenesis in various BMPs. BMP9 phosphorylates the transcription factor smad1, smad5 or smad8. These phosphorylated $\mathrm{r}$-smad factors bind to smad4 and localize to the nucleus to promote the expression of osteogenic genes $[10,11]$. In addition, some factors or signals, such as $\mathrm{Wnt} / \beta$-catenin, ATRA and EGF, interact with BMP9 to enhance the BMP9-induced osteogenic differentiation of MSCs [3, 4, 12].

Melatonin ( $\mathrm{N}$-acetyl-5-methoxytryptamine) is a hormone secreted by the pineal gland that affects circadian rhythm, regulates the sleep-wake cycle, inhibits tumour growth and regulates immunity [13]. In most mammals, including humans, melatonin receptors have two subtypes, MT1 and MT2, which belong to the G proteincoupled receptor family [14, 15]. Melatonin exerts its physiological regulation mainly through receptors distributed in the hypothalamus (PT) and suprachiasmatic $(\mathrm{SCN})$, and peripheral tissues, such as the nervous system, retina, immunity system, reproductive system and endocrine system tissues, also show a distribution of these receptors. Studies have shown that melatonin may have a regulatory effect on bone metabolism: promoting the osteogenic differentiation of BMSCs while inhibiting adipogenic differentiation [16, 17]; inhibiting osteoclast formation and activation, thereby inhibiting bone resorption [18]; and activating the antioxidant defence system to maintain the self-renewal and differentiation of BMSCs after long-term passage [19]. Additionally, the expression of melatonin receptors was also found in MSCs. It is currently believed that the osteogenic effects of melatonin are mainly mediated through the MT2 receptor, and the mechanisms involved include TGF- $\beta$ [20], the Wnt/ $\beta$-catenin pathway [15] and the MAPK signalling pathway [21]. It has been found that in pituitary AtT20 cells and rat granulosa cells, melatonin promotes the expression of BMP4 or BMP6 and phosphorylation of Smad1/5/8 downstream of BMPs $[22,23]$. The melatonin-induced expression of osteogenic markers, such as BMP4 or BMP2, was also found in mouse pre-myoblast cell lines, $\mathrm{C} 2 \mathrm{C} 12$ cells and human dental pulp stem cells $[24,25]$. However, whether this effect of melatonin on BMPs is also present in MSCs and how the interaction between melatonin and BMPs occurs during the osteogenic differentiation of MSCs is still unclear, so further research is needed.

The specific purpose of this study was to investigate the effects of melatonin on the BMP9-induced osteogenic differentiation of MSCs and to reveal the underlying molecular mechanisms of this effect. Our results suggest that melatonin combined with BMP9 promotes osteogenic differentiation by activating the AMPK/ $\beta$-catenin signalling pathway. Therefore, melatonin may provide another potentially effective option for enhancing BMP9-induced osteogenesis in bone marrow mesenchymal stem cells. This effect has important clinical significance for promoting the application of MSCs in the field of bone tissue engineering and research, treatment and prevention of the pathogenesis of bone metabolism diseases, such as osteoporosis, and fracture healing.

\section{Methods \\ Cell culture and chemicals}

C3H10T1/2 cells were purchased from Shanghai Institutes for Biological Sciences (Shanghai, China), and the cells were cultured in Dulbecco's modified Eagle's medium (DMEM) with 10\% foetal bovine serum (FBS), $100 \mathrm{U} / \mathrm{ml}$ penicillin and $100 \mathrm{mg} / \mathrm{ml}$ streptomycin. The culture environment was $37^{\circ} \mathrm{C}$ in $5 \% \mathrm{CO}^{2}$. Melatonin was diluted to $100 \mathrm{mM}$ as storage concentrations in DMSO. DMSO was used as a solvent control. Unless otherwise indicated, all chemicals were purchased from Sigma-Aldrich (St. Louis, MO, USA).

\section{Alkaline phosphatase (ALP) activity and staining}

The total cellular protein concentration of the sample was determined using a BCA protein assay (Beyotime, China). The activity of ALP was quantitatively determined by modified Great EscAPe SEAP Chemiluminescence Assay (BD Clontech, USA) as previously described. According to the definition of enzyme activity, the alkaline phosphatase activity in the sample was calculated. Qualitative detection of ALP activity was performed using a BCIP/NBT alkaline phosphatase staining assay kit (Beyotime, China).

\section{Alizarin red $\mathrm{S}$ staining}

Alizarin red $\mathrm{S}$ staining was carried out as described above [8-10]. After the corresponding treatment, C3H10T1/2 cells were cultured in the presence of $50 \mu \mathrm{g} /$ $\mathrm{ml}$ ascorbic acid, $100 \mathrm{nM}$ dexamethasone and $10 \mathrm{mM} \beta$ glycerophosphate. The medium was changed every 3 days. After 21 days of culture, the medium was removed, the cells were washed twice with PBS and fixed at room temperature for $15 \mathrm{~min}$ using $4 \%$ paraformaldehyde, and the cells were washed twice more with PBS. The fixed cells were incubated with $2 \%$ Alizarin red S solution (Sigma-Aldrich) for $10 \mathrm{~min}$ at room temperature and then washed thoroughly in PBS. Calcium mineral deposition staining was observed by light microscopy (Leica DMI 3000B, Germany). 


\section{Immunohistochemical (IHC) staining}

After 14 days of the corresponding treatment, C3H10T1/2 cells were fixed with $4 \%$ paraformaldehyde and washed with PBS. The cells were permeabilized with $0.1 \%$ Triton-X (Sigma, USA) and blocked with 10\% BSA (Beyotime, China) to reduce non-specific staining. Subsequently, the cells were incubated with anti-osteocalcin (OCN) antibody (sc30045, Santa Cruz Biotechnology) and anti-osteopontin (OPN) antibody (ab91655, Abcam) at $4{ }^{\circ} \mathrm{C}$ overnight. The cells were washed three times with DPBS and incubated with a biotin-labelled secondary antibody (Santa Cruz Biotechnology, USA) for 20 $\min$ at $37^{\circ} \mathrm{C}$. The cells were washed three times with DPBS, and a streptavidin-HRP conjugate was added to the cells to incubate for $20 \mathrm{~min}$ at $37^{\circ} \mathrm{C}$. The presence of the expected protein was observed by diaminobenzidine (DAB) staining and examined under a microscope. Control IgG staining was used as a negative control.

\section{Western blot analysis}

The cells were lysed by ice-cold RIPA lysis buffer (Biyuntian, China) containing protease inhibitors, and the protein concentration was quantified using a BCA protein assay kit (Biyuntian). Each group of proteins was denatured by boiling, and then sodium dodecyl sulfatepolyacrylamide gel electrophoresis (SDS-PAGE) was used to separate the proteins. Subsequently, the proteins were transferred to a polyvinylidene fluoride (PVDF) membrane (Millipore, USA) by electrophoresis. Then, the membranes were blocked in 5\% skim milk for $1 \mathrm{~h}$ and incubated overnight at $4{ }^{\circ} \mathrm{C}$ in diluted primary antibody. Finally, images of the target strip were developed using a Western Chemiluminescent HRP Substrate Kit (Millipore, USA). Image Lab software (Bio-Rad, USA) was used for semi-quantitative analysis. Primary antibodies directed against AMPK (D5A2 for monoclonal antibody, Cell Signaling Technology), p-AMPK (40H9 for monoclonal antibody, Cell Signaling Technology), $\beta$ catenin (polyclonal antibodies, Cell Signaling Technology), p- $\beta$-catenin (polyclonal antibodies, Cell Signaling Technology), p-Smad-1/5/8(D5B10 for monoclonal antibody, Cell Signaling Technology), Smad-1/5/8 (N-18 for monoclonal antibody, Santa Cruz Biotechnology) and GADPH (6C5 for monoclonal antibody, Beyotime) were used.

\section{Transient transfection with small interfering RNAs}

For RNA interference against AMPK $\alpha 1 / 2$ and CTNNB1, siRNA duplexes were synthesized, corresponding to AMPK $\alpha 1 / 2$ (target sequence: 5'-AAGAGAAGCAGAAG CACGACG-3'), CTNNB1 (sense: 5'-AUCACAGAUG UUGAAACAUTT- $3^{\prime}$ and antisense: 5'-AUGUUU CAACAUCUGUGAUGG-3') and control (5'-AAGCCG GTATGCCGGTTAAGT-3'). The cells were transfected with $25 \mathrm{nM}$ siRNA using Lipofectamine 2000 transfection reagent (Invitrogen, USA) according to the manufacturer's instructions and subsequently treated at 2 days after transfection.

\section{Immunofluorescence staining}

Cells cultured in 24-well plates were fixed with $4 \%$ paraformaldehyde for $20 \mathrm{~min}$ at room temperature and then permeabilized using $0.5 \%$ Triton X-100 for $20 \mathrm{~min}$. The fixed cells were then blocked in 5\% BSA for $2 \mathrm{~h}$ and incubated overnight at $4{ }^{\circ} \mathrm{C}$ in a suitably diluted primary antibody against p-Smad1/5/8 (1:500, Cell Signaling). After washing with PBS, the cells were incubated with Alexa Fluor 647-labelled goat anti-rabbit IgG $(\mathrm{H}+\mathrm{L})$ antibody (1:500 dilution; Beyotime, China) at room temperature in the dark for $1 \mathrm{~h}$. The cells were then incubated with DAPI for 10 min and sealed with sealing liquid containing an anti-fluorescence quencher, and images were captured on a Leica DMI 3000B inverted fluorescence microscope.

\section{Foetal limb explant culture}

Foetal limbs were obtained by dissecting mouse embryos (E18.5) under sterile conditions and culturing in DMEM containing $0.5 \%$ BSA, $50 \mu \mathrm{g} / \mathrm{ml}$ ascorbic acid, $1 \mathrm{mM} \beta$ glycerophosphate and $100 \mathrm{mg} / \mathrm{ml}$ penicillin and streptomycin (Sigma) at $37^{\circ} \mathrm{C}$ in $5 \% \mathrm{CO}_{2}$. After $24 \mathrm{~h}$ of in vitro culture, the limbs were treated accordingly. The medium was changed every 3 days. After 10 days of culture, calcein (100 mM, Sigma, USA) was added to the medium. After 12 days, the skin and muscle were removed, and new bone formation was assessed using fluorescence microscopy and histology. Each group includes at least five limb explants.

\section{C3H10T1/2 implantation and micro-computed tomographic $(\mu \mathrm{CT})$ analysis}

Male athymic nude mice (4 weeks old) were obtained from Shanghai Laboratory Animal Research Center (Shanghai, China). C3H10T1/2 cells were treated with AdBMP9, AdGFP and melatonin alone or in combination, and the infection efficiency was confirmed by fluorescence microscopy at $24 \mathrm{~h}$ later. After 7 days of treatment, the cells were harvested for subcutaneous injection $\left(5 \times 10^{6}\right.$ cells per injection) into the flanks of athymic nude $(\mathrm{nu} / \mathrm{nu})$ mice ( 5 animals per group). Then, intrathecal injection was performed with melatonin (5 $\mathrm{mg} / \mathrm{kg} /$ day) or PBS, and after 5 weeks, the animals were euthanized, and the specimen was removed and fixed. Scanning and histological evaluation of ectopic bone were performed using a high-resolution $\mu \mathrm{CT}$ system (SkyScan, Belgium) and analysis of the data using $\mu \mathrm{CT}$ analyser software (SkyScan, Belgium). All experiments were performed in accordance with the guidelines for 
animal experiments of the Xinhua Hospital Ethics Committee.

\section{Histological evaluation}

The removed tissues were fixed with $4 \%$ paraformaldehyde, then decalcified and dehydrated with paraffin embedded. Serial sections of paraffin-embedded samples were stained with haematoxylin and eosin (H\&E) or Masson's trichrome stain as previously described $[26,27]$.

\section{RNA extraction and real-time reverse transcription} polymerase chain reaction (real-time RT-PCR) analysis

The cells were seeded in 6-well plates, and treated with AdBMP9, AdGFP and melatonin alone or in combination for 2 days to extract total cellular RNA and perform reverse transcription. The $\mathrm{qPCR}$ reaction was carried out using a SYBR Green Premix Ex TaqTM kit (Takara, Japan). The reaction conditions were as follows: $95^{\circ} \mathrm{C}$ for $5 \mathrm{~s} ; 60{ }^{\circ} \mathrm{C}$ for 30 s; 40 cycles. GAPDH was used as the internal reference gene, and the relative quantification was performed by the $\Delta \Delta \mathrm{Ct}$ method (Comparative Delta-delta $\mathrm{Ct}$ ).

\section{Isolation of mouse bone marrow mesenchymal stem cells} (BMSCs)

Primary MSCs were collected from the bone marrow of C57 mice using the method of Soleimani et al. [28]. The 8week-old mouse was sacrificed by cervical dislocation, and then the femur and tibia were separated. The bone marrow was washed out with Dulbecco's modified Eagle's medium low glucose (DMEM LG, SH30021.01, Hyclone) with a 1$\mathrm{ml}$ syringe until the bones turned white. The bone marrow extract was filtered through a 70- $\mu \mathrm{m}$ cell strainer and pelleted at $1000 \mathrm{rpm}$ for $5 \mathrm{~min}$. The cell pellet was resuspended in $15 \mathrm{~mL}$ DMEM LG containing 10\% FBS and 100 $\mathrm{mg} / \mathrm{ml}$ Pen-Strep and inoculated into a T-75 $\mathrm{cm}^{2}$ flask. The solution was changed every 3 days. After the cell fusion degree reaches $80-90 \%$, the cells are passaged, and the fourth generation can be used for experiments.

\section{Statistical analysis}

All data are expressed as the means \pm standard error (SE). The experiments were performed at least three times to ensure reproducibility. Statistical differences between the two groups were determined using one-way analysis of variance (ANOVA) or Student's $t$ test. A $P$ value $<0.05\left({ }^{*}\right)$ was considered statistically significant.

\section{Results}

Melatonin synergizes with BMP9 to induce the ALP activity of $\mathrm{C} 3 \mathrm{H} 10 \mathrm{~T} 1 / 2$ cells

To explore the effects of melatonin and BMP9 in synergistically inducing the osteogenic differentiation of $\mathrm{MSC} /$ C3H10T1/2 s, we used a recombinant adenovirus expressing human BMP9 as described above and demonstrated that this recombinant adenovirus is capable of efficiently transducing C3H10T1/2 cells (Fig. 1a). We treated C3H10T1/2 cells with $0 \mathrm{nM}$ (control), $100 \mathrm{nM}, 1 \mu \mathrm{M}$, $10 \mu \mathrm{M}$ and $100 \mu \mathrm{M}$ melatonin for 3, 5 and 7 days to determine the effect of melatonin on the osteogenic differentiation of C3H10T1/2 cells. The ALP activity of C3H10T1/ 2 cells increased with increasing melatonin dose and $100 \mu \mathrm{M}$ melatonin was able to induce ALP activity to the greatest extent (Fig. 1b, d), which was selected for subsequent experiments. Next, we used melatonin $(100 \mu \mathrm{M})$ and BMP9 alone or in combination to stimulate C3H10T1/2 cells. The ALP activity assay showed that BMP9 induced ALP activity earlier and stronger than melatonin stimulation. The combination of both melatonin and BMP9 can further enhance ALP activity (Fig. 1c, e). In addition, we performed the same experiment in primary MSC cells, and the results were similar to those in C3H10T1/2 cells, and the combination of melatonin and BMP9 further enhanced ALP activity (Fig. 1f). In summary, the data obtained indicate that melatonin can synergize with BMP9 to induce ALP activity in C3H10T1/2 cells.

\section{The combination of melatonin and BMP9 enhances the late osteogenic marker expression and matrix mineralization of $\mathrm{C} 3 \mathrm{H} 10 \mathrm{~T} 1 / 2$ cells}

To further confirm these results, we analysed the effect of melatonin on BMP9-induced late osteogenic markers. The results of Alizarin red $\mathrm{S}$ staining showed that the formation of calcium mineral deposits in C3H10T1/2 cells stimulated by melatonin and BMP9 was significantly increased compared with BMP9 infection alone, and the matrix mineralization was obvious (Fig. 2a). Immunohistochemical staining showed that melatonin significantly enhanced the expression of the BMP9-induced late osteogenic markers osteocalcin (Fig. 2b) and osteopontin (Fig. 2c). In addition, we examined the expression of osteogenesis-related genes RUNX2, Osterix, Col1 and BMP2 mRNA. The results showed that melatonin combined with BMP9 significantly increased the expression levels of RUNX2, Osterix and Col1 mRNA, while the increase of BMP2 mRNA expression levels was not statistically significant (Fig. 2d). In addition, we also performed Alizarin red $S$ staining in primary MSC cells, and the results were similar to those in C3H10T1/2 cells, the combination of melatonin and BMP9 further enhanced the formation of calcium mineral deposits in MSCs (Fig. 2e). Based on these results, we conclude that melatonin signalling can synergize with BMP9-induced osteogenic signalling in C3H10T1/2 cells.

\section{Melatonin promotes BMP9-induced osteogenesis of embryonic limbs in vitro}

Next, we examined the effects of melatonin and BMP9 on bone development through foetal limb culture. E18.5 


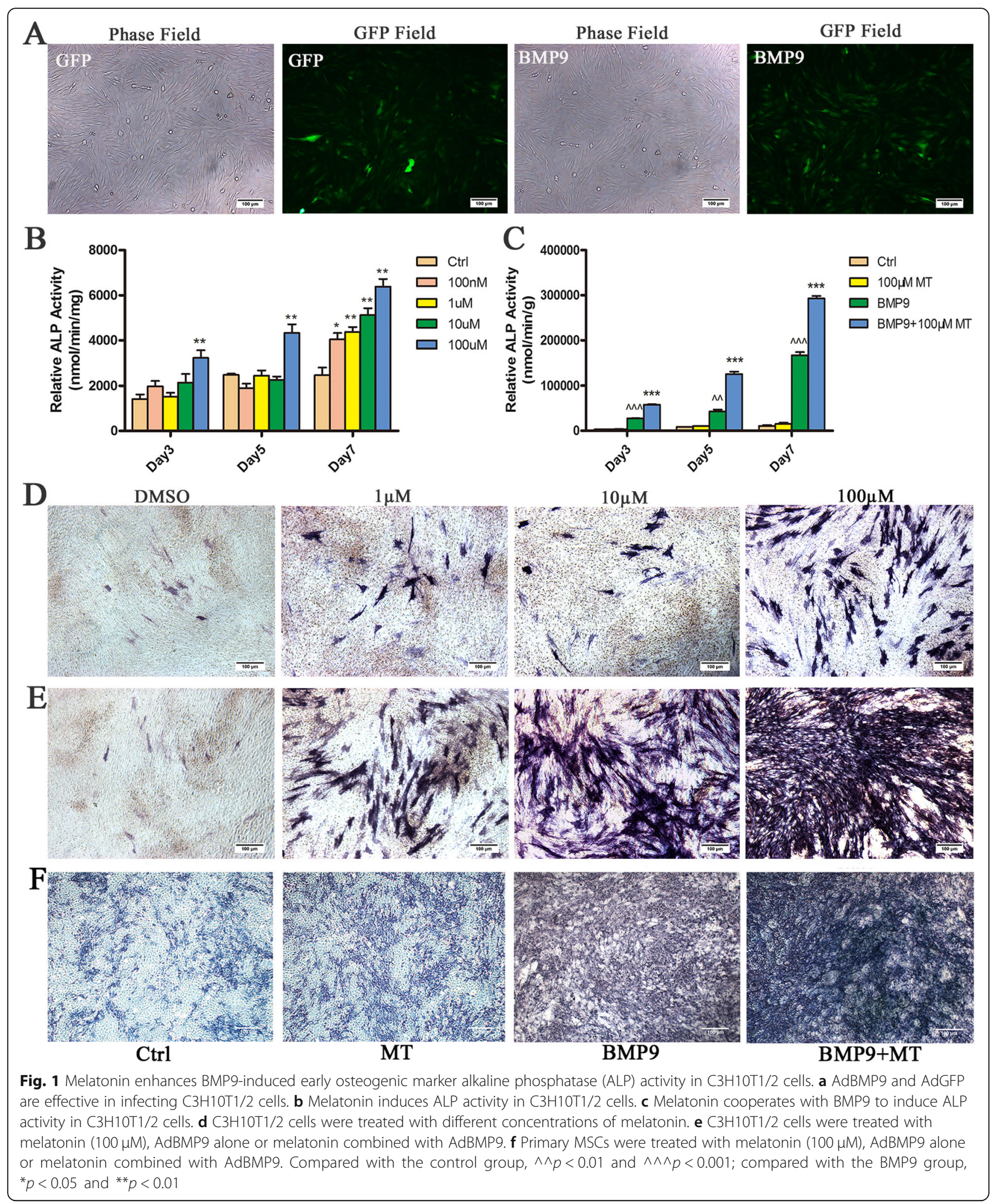

mouse embryo limbs ( $n=5$ per group) were obtained, and then the isolated limbs were infected with AdBMP9 or AdGFP in the presence or absence of melatonin $(100 \mu \mathrm{M})$. After 10 days, the fluorescent dye calcein was added to the medium to mark new bone formation. We found that the BMP9 group and melatonin + BMP9 group were more active in bone composition and showed faster bone development than did the control 


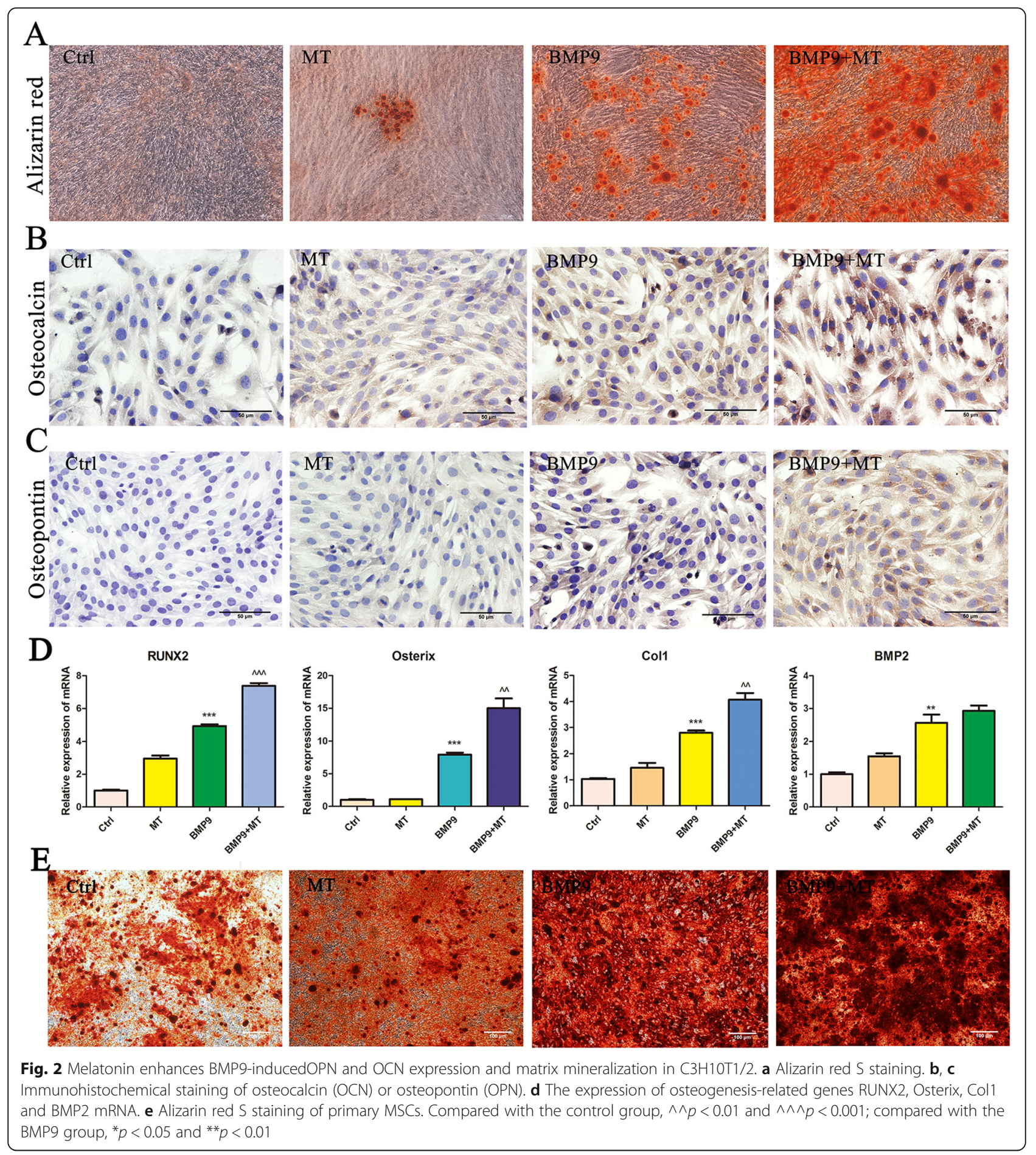

group. Relative to the BMP9 group alone, the melatonin + BMP9 group showed higher fluorescence intensity, indicating that new bone formation was more active (Fig. 3a). Next, we further confirmed the above results through histological analysis. Compared with the GFP control group, melatonin or BMP9 stimulation alone increased the area of the proliferation zone, while co-treatment further increased the proliferation zone (Fig. 3b, c). Compared with the groups treated with melatonin or BMP9 alone, the melatonin + BMP9 group had thicker trabecular matrix (Fig. 3d). These results indicate that melatonin can increase the BMP9-induced endochondral ossification of foetal limb culture. 


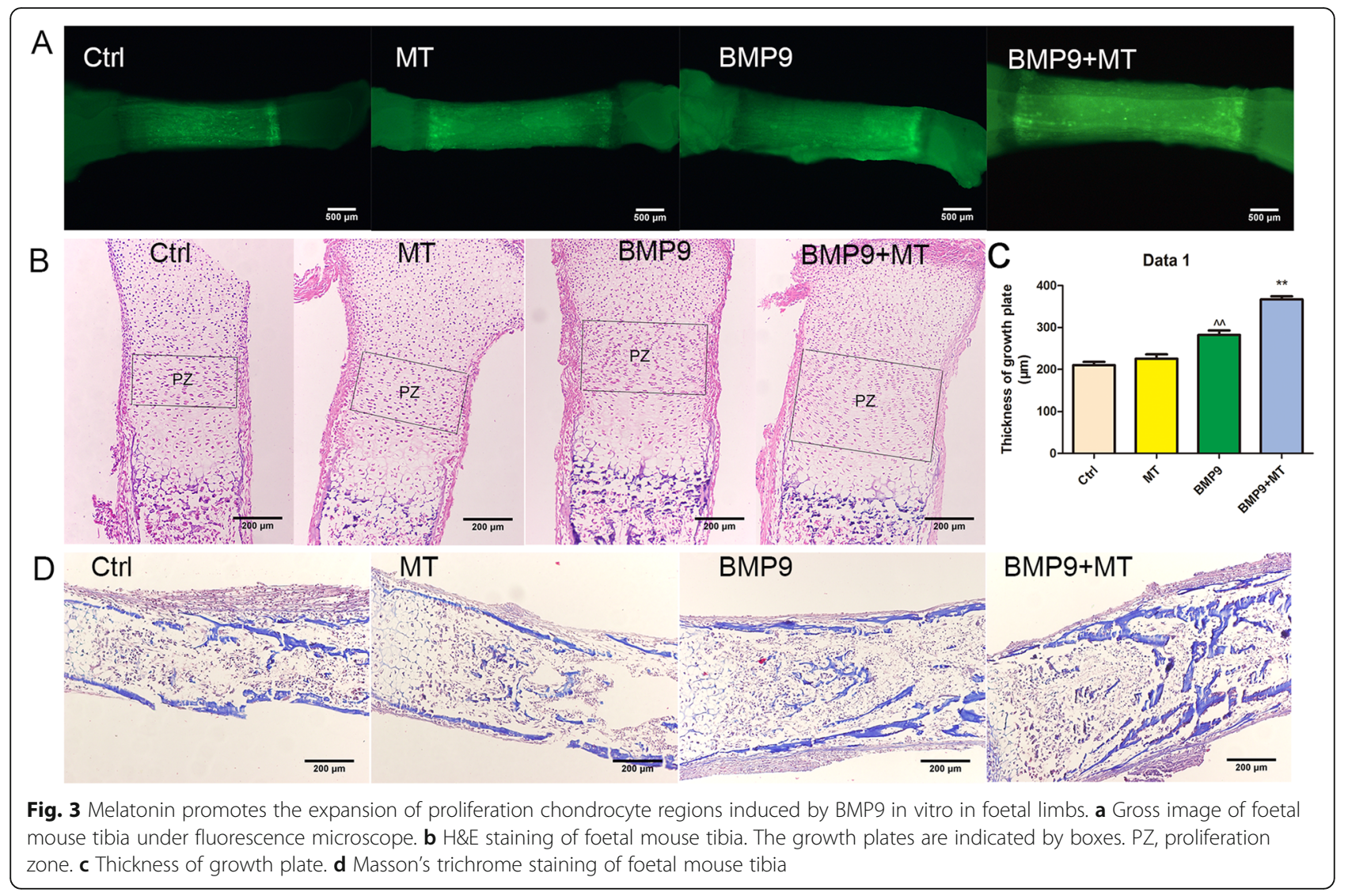

Melatonin enhances BMP9-induced ectopic bone formation To further confirm the role of melatonin in the BMP9induced osteogenic differentiation of C3H10T1/2 cells, we performed in vivo stem cell transplantation. We pretreated C3H10T1/2 cells with melatonin and/or BMP9 for 7 days, and the cells were harvested and injected subcutaneously into athymic nude mice, and the mice were treated with melatonin or saline subcutaneous injection. At week 6, the BMP9 group and the melatonin/BMP9 group formed ectopic bone, while the control group and the melatonin group did not have bone formation. Micro-CT scan data showed that melatonin/BMP9 treatment resulted in increased bone mass and relative bone density compared to the BMP9 group (Fig. 4b, c). The melatonin/BMP9 group formed a more mature and thicker trabecular matrix (Fig. 4d). Masson's trichrome staining showed that melatonin enhanced the BMP9induced mineralized bone matrix (Fig. 4e). Taken together, these data indicate that melatonin increases the BMP9induced osteogenic differentiation of C3H10T1/2 cells.

\section{Melatonin promotes the BMP9-induced activation of its downstream signalling pathways in regulating the osteogenic differentiation of C3H10T1/2 cells}

The BMP signalling pathway plays a key role in the osteogenic differentiation of MSCs. BMP9 is considered to be one of the most potent factors in inducing the osteogenic differentiation of MSCs [4]. Studies have shown that melatonin can induce the osteogenic differentiation of MSCs and increase the expression of BMP4 and BMP6 in other cells $[22,23]$. The results of our previous experiments show that melatonin can significantly enhance the osteogenic differentiation of BMP9-induced MSCs, and then we tried to determine the mechanism of interaction between melatonin and BMP9 signalling in MSCs. We found that compared to BMP9 alone, the combination of melatonin and BMP9 resulted in a significant increase in BMP9 expression and the phosphorylation of its downstream protein Smadl/5/8 in C3H10T1/2 cells (Fig. 5a, b). However, Smadl $/ 5 / 8$ total protein levels did not change significantly between groups. The results of immunofluorescence further confirmed that melatonin and BMP9 co-treatment significantly upregulated Smad1/5/8 phosphorylation and promoted the localization of phosphorylated Smad1/5/8 to the nucleus (Fig. 5c). These data indicate that melatonin may at least partially promote the BMP9-induced activation of BMP9/Smadl/5/8 signalling in C3H10T1/2 cells.

\section{Melatonin synergizes with BMP9 to promote the osteogenic differentiation of $\mathrm{C} 3 \mathrm{H} 10 \mathrm{~T} 1 / 2$ cells via the AMPK/ $\beta$-catenin pathway}

Studies have shown that adenosine-activated protein kinase (AMPK), an energy-sensing kinase, can regulate 


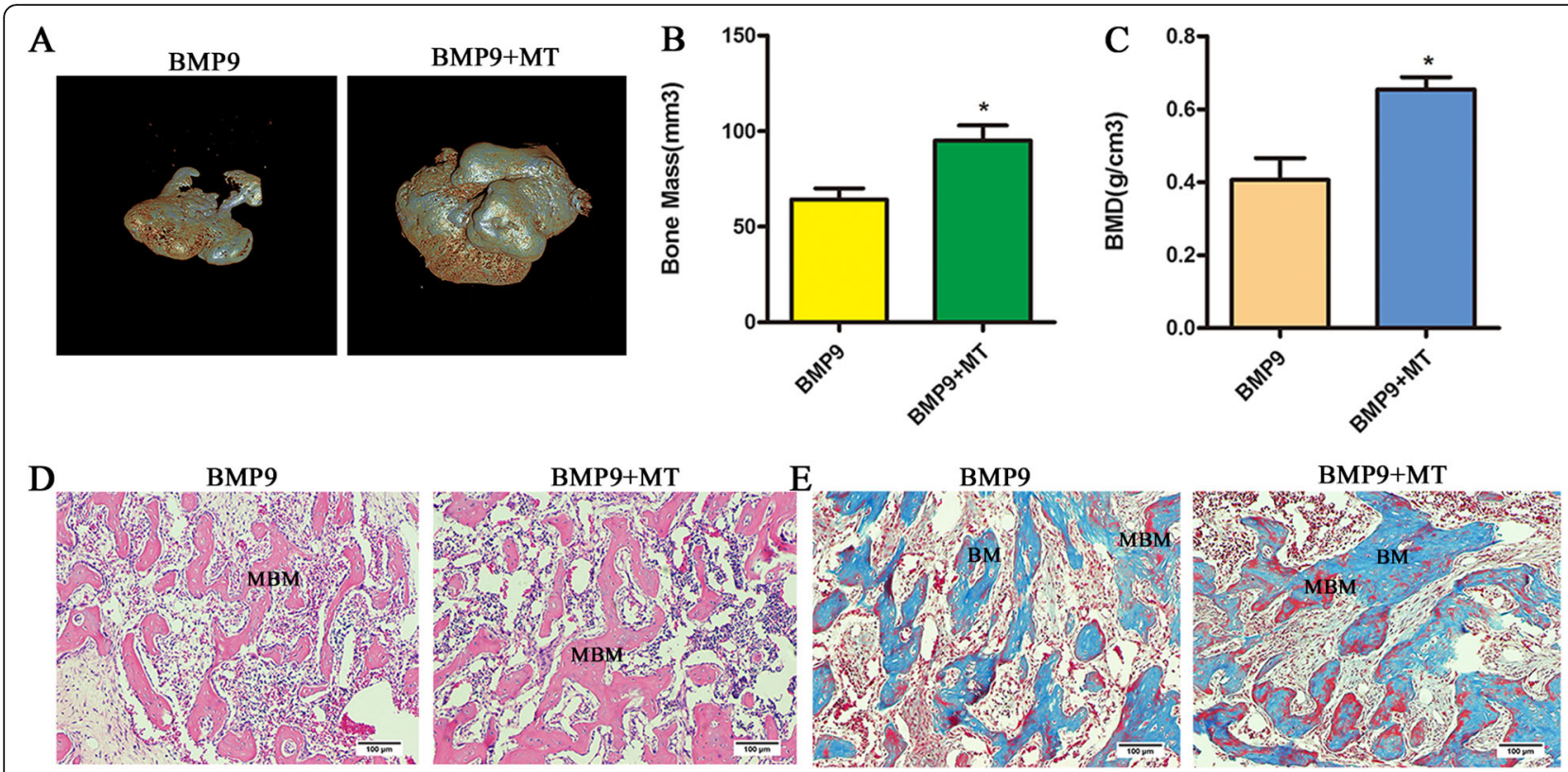

Fig. 4 Melatonin enhances BMP9-induced ectopic bone formation. a Scanned image of ectopic bone using micro-CT. b, c Analysis of bone mass and relative bone density. ${ }^{*} p<0.05$. $\mathbf{d}$, e Histological analysis of specimens (H\&E staining and Masson's trichrome staining). BM, bone matrix; MBM, mineralized bone matrix

A

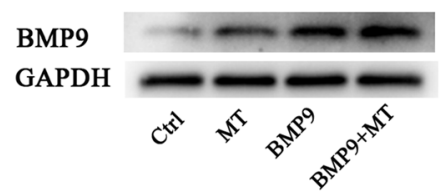

C
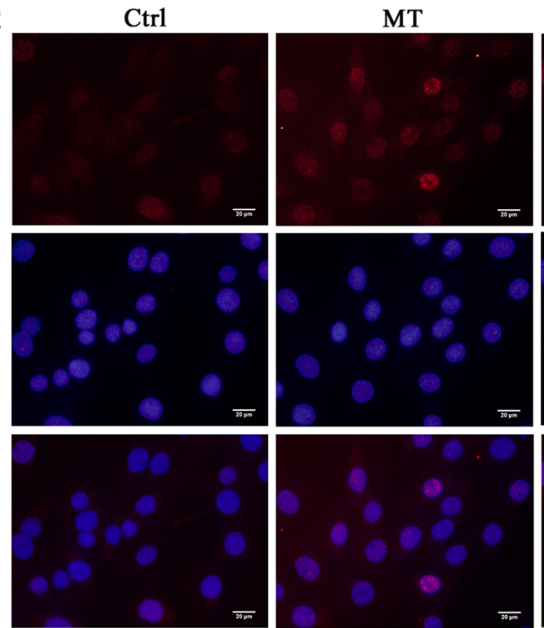

B

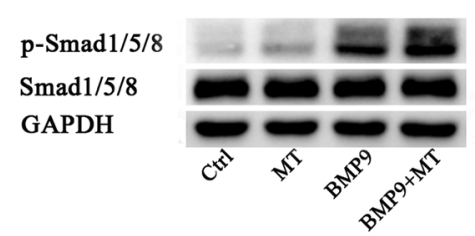

BMP9
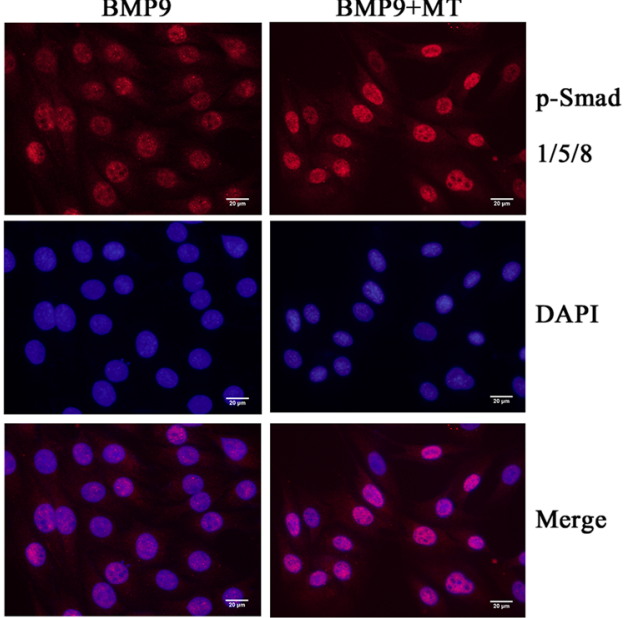

Fig. 5 Melatonin enhances the BMP9 signalling pathway in the regulation of C3H10T1/2 osteogenic differentiation. $\mathbf{a}$, $\mathbf{b}$ Melatonin enhances BMP9 expression and the phosphorylation of its downstream protein Smad1/5/8 in C3H10T1/2 cells. c p-Smad1/5/8 is transferred from the cytoplasm to the nucleus 
bone metabolism by activating beta-catenin [29]. Melatonin can protect the osteogenic differentiation function of MSCs by promoting the activation of AMPK or $\beta$ catenin $[20,30]$. In multiple sources of MSCs, BMP9 and $\mathrm{Wnt} / \beta$-catenin signalling synergistically promote osteogenic differentiation, and the knockdown of $\beta$ catenin eliminates the elevation of BMP9-induced osteogenic marker ALP [4, 12]. Therefore, we examined whether melatonin and BMP9 have an effect on the activation of AMPK $\alpha$ and $\beta$-catenin. Although there were no significant changes in AMPK $\alpha$ and $\beta$-catenin protein levels in all groups, the phosphorylation of AMPK $\alpha$ at Thr172 and the phosphorylation of $\beta$-catenin at Ser552 were significantly increased (Fig. 6a). To further confirm the role of AMPK $\alpha / \beta$-catenin in melatonin- and BMP9induced osteogenic differentiation, we treated C3H10T1/ 2 cells with AMPK $\alpha$ siRNA or $\beta$-catenin siRNA with a good silencing effect (Fig. 6c, d). Pretreatment with siAMPK $\alpha$ blocked the $\beta$-catenin phosphorylation induced by co-treatment with melatonin and BMP9 (Fig. 6b). Additionally, pretreatment with siAMPK $\alpha$ or si $\beta$-catenin significantly inhibited the increase in ALP, phosphorylation of Smad1/5/8 and expression of BMP9 caused by the synergistic induction of melatonin and BMP9 (Fig. 6e-j). This finding suggests that the AMPK/ $\beta$-catenin pathway is at least partially involved in melatonin/BMP9-induced osteogenic differentiation and that AMPK may play a role, at least in the upstream signalling of $\beta$-catenin in MSCs.

In summary, melatonin and BMP9 signalling show crosstalk between the following pathways. Melatonin/ BMP9 can directly upregulate bmp9 and its downstream targets and promote the transfer of $\mathrm{p}-\mathrm{Smad} 1 / 5 / 8$ from the cytoplasm to the nucleus, which is more significant than melatonin or BMP9 alone. By activating the AMPK $\alpha / \beta$-catenin signalling pathway, melatonin/BMP9 synergizes and leads to efficient bone formation.

\section{Discussion}

In this study, we explored the possible synergistic relationship between melatonin and BMP9 in inducing the osteogenic differentiation of mesenchymal stem cell line C3H10T1/2 cells. The results indicate that melatonin can synergize with BMP9 to induce the osteogenic differentiation of $\mathrm{C} 3 \mathrm{H} 10 \mathrm{~T} 1 / 2$ cells through the AMPK/ $\beta$ catenin pathway. Currently, the role and mechanism of melatonin on the skeletal system has not been fully clarified, and the role of the melatonin and BMP pathway interaction in the process of MSC osteogenic differentiation has rarely been reported. The mechanism of action between the two is very necessary.

BMPs play an important regulatory role in cell proliferation and differentiation during embryonic development and are key signalling pathways regulating the osteogenic differentiation of mesenchymal stem cells [7]. The interruption of BMP signalling leads to various bone and extra-bone abnormalities [31]. BMPs have the ability to regulate the multilineage-specific differentiation of MSCs. BMP2, BMP4, BMP6, BMP7 and BMP9 can effectively induce adipogenesis and osteogenic differentiation in vitro and in vivo, and BMP-induced osteogenesis or adipogenic differentiation has been shown to be mutually exclusive [12]. BMP9 is one of the most potent factors in the BMP family to induce the osteogenic differentiation of mesenchymal stem cells in vivo and in vitro, which have the ability to form bone alone. However, many signalling pathways with different functions have been found to play a role in BMP9-mediated osteogenesis, such as Wnt/ $\beta$-catenin, ATRA and IGF $[3,4,32]$.

Studies have shown that melatonin may have a positive effect on bone metabolism, but its osteogenesis mechanism remains unclear. Melatonin has widely distributed receptors, including $\mathrm{G}$ protein-coupled melatonin receptors (MT1/MT2), nuclear receptors (ROR/RZR receptors), calmodulin and mitochondria. Among these receptors, the G protein receptor (MT1/MT2) is the classical pathway for melatonin [33]. For perimenopausal women, nocturnal melatonin causes a time-dependent decrease in the ratio of serum osteoclasts and osteoblasts [34]. Similarly, as age increases, melatonin levels decrease. Bone mineral density (BMD) in the femoral neck increases after treatment with melatonin in postmenopausal women [35]. Mechanistically, although melatonin has no significant effect on the proliferation of MSCs $[36,37]$, melatonin has a regulatory effect on mesenchymal stem cell differentiation, which can promote osteogenic and chondrogenic differentiation [38], inhibit lipid differentiation [36] and maintain the self-renewal and differentiation characteristics of MSCs [36]. Melatonin can also play a protective role in osteogenic differentiation disorders and ageing-related osteoporosis by activating the antioxidant defence system, increasing bone formation or reducing bone resorption [13, 30, 39]. Extracellular matrix (ECM) regulates the physiological function of hormones by providing binding sites and modulating downstream signalling pathways. The interaction of melatonin with ECM deposited by natural cells can protect the osteogenic differentiation ability of MSCs [40]. Therefore, melatonin may provide a new option for the treatment of osteoporosis and fracture [19].

Previous studies have shown that melatonin promotes the expression of BMP4 or BMP6 in pituitary AtT20 cells or rat granulosa cells and the phosphorylation of Smad1/5/8 downstream of BMPs [22, 23]. Similarly, melatonin can activate osteogenic markers, such as Runx2, OCN and BMP2 and BMP4, in pre-osteoblast MC3T3-E1 cells in a dose-dependent manner [20]. Therefore, we speculate that melatonin may induce 


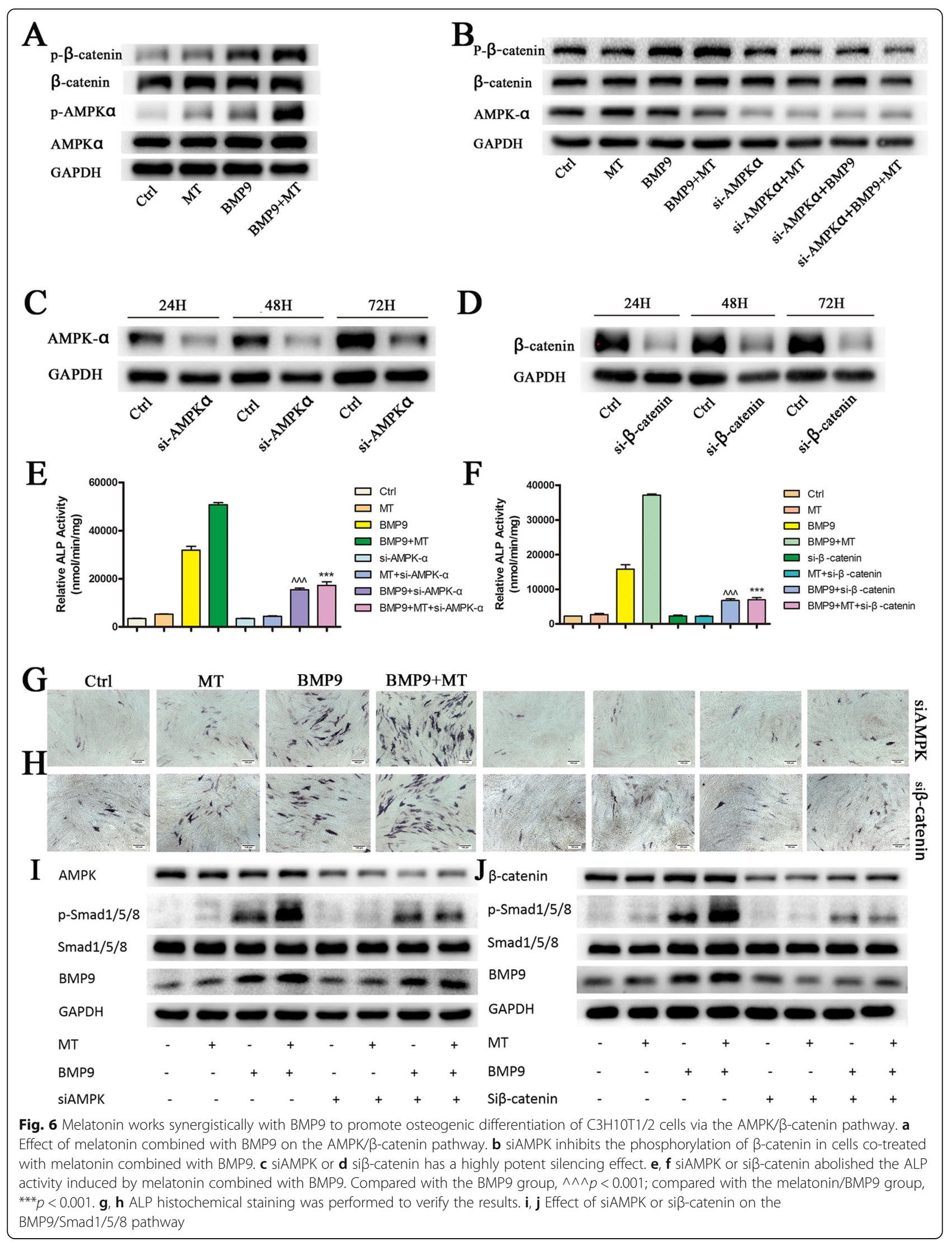


BMP9 expression during the osteogenic differentiation of $\mathrm{C} 3 \mathrm{H} 10 \mathrm{~T} 1 / 2$ cells, and there may be a mutual promoting effect between these two factors. Our results confirm this idea, as melatonin induces BMP9 expression in C3H10T1/2 cells and enhances BMP9-induced Smad1/ 5/8 phosphorylation and Smad1/5/8 nuclear translocation. Although both melatonin and BMP9 were able to induce osteogenic marker expression alone, the combination of the two stimuli significantly enhanced ALP activity as well as OCN and OPN expression.

The osteogenic synthesis of collagen for bone modelling and bone remodelling requires the consumption of large amounts of adenosine triphosphate (ATP) [41]. Disorders in circadian rhythms can alter a variety of proteins that regulate glucose balance and/or energy metabolism in the body [42]. Melatonin may play a role in the regulation of energy metabolism by affecting the circadian rhythm [43, 44]. Leptin and adiponectin are adipokines that are closely related to glucose and lipid metabolism and energy balance. Circadian rhythm disorders can interfere with the synthesis and secretion of leptin and adiponectin, and melatonin supplementation normalizes the expression and secretion patterns of these two adipokines [45]. AMPK is a key regulator of energy metabolism and is regulated by a variety of factors, such as leptin, adiponectin and resistin [46, 47]. Emerging evidence suggests that AMPK regulates cell differentiation in addition to its role in metabolic processes [48, 49]: AMPK promotes osteogenesis and inhibits lipogenesis in MC3T3-E1 cells [50]; and active AMPK can directly phosphorylate RUNX2 and promote osteogenesis [51]. Therefore, we speculate that melatonin may achieve synergistic osteogenesis with BMP9 by activating AMPK. This idea is consistent with our findings that melatonin combined with BMP9 treatment increases phosphorylated AMPK levels.

Both BMP and Wnt signalling are involved in the regulation of osteoblast differentiation and bone formation. In MSCs, Wnt3a and BMP9 regulate overlapping but distinct downstream target gene expression [52, 53], suggesting that crosstalk may exist between their induced osteogenic signalling pathways, as demonstrated by subsequent studies $[4,54]$. Crosstalk between AMPK and $\mathrm{Wnt} / \beta$-catenin signalling was confirmed in both in vitro and in vivo experiments $[55,56]$. AMPK phosphorylates $\beta$-catenin at Ser 552, stabilizes $\beta$-catenin and enhances $\beta$-catenin/TCF-mediated transcription, thereby regulating cell differentiation and developmental signalling pathways $[46,57]$. Therefore, we propose that $\beta$ catenin may act downstream of AMPK and play a role in the osteogenic differentiation induced by melatonin in combination with BMP9. To test this hypothesis, we examined changes in AMPK/ $\beta$-catenin in melatonin/ BMP9-induced bone formation. The results indicate that melatonin and BMP9 have a synergistic effect on the activation of $A M P K / \beta$-catenin signalling. The knockout of AMPK or $\beta$-catenin abolishes the stimulatory effect of melatonin on BMP9-induced alkaline phosphatase activity. Therefore, we propose that melatonin combined with BMP9 may be an effective treatment for bone metabolic diseases, such as osteoporosis, and fracture healing.

However, our research also has some limitations. Studies have shown that the activation of the AMPK kinase complex induces mitochondrial division, increases apoptosis and reduces the viability of MSCs. Knocking out AMPK may help reduce MSC damage in patients with myelodysplastic syndrome [57]. Osteogenic differentiation is a multifactorial regulation process, and the molecular mechanism of the AMPK/ $\beta$-catenin pathway in osteogenic differentiation of C3H10T1/2 cells induced by melatonin and BMP remains to be further studied.

\section{Conclusions}

In summary, we investigated the effects of melatonin on BMP9-induced early and late osteogenic markers. Mechanistically, we found that this process may be at least partially mediated by the AMPK/ $\beta$-catenin pathway, and silencing AMPK or $\beta$-catenin can effectively attenuate BMP9-induced osteogenesis. Therefore, the interaction between melatonin and the BMP9 pathway may play an important role in regulating the osteogenic differentiation of MSCs. Exploring the combination of melatonin and BMP9 provides a new treatment for bone metabolic diseases, such as osteoporosis.

\section{Abbreviations}

MSCs: Mesenchymal stem cells; BMP9: Bone morphogenetic protein (BMP)-9; MT: Melatonin; ALP: Alkaline phosphatase; OPN: Osteopotin;

OCN: Osteocalcin

\section{Acknowledgements}

We would like to thank Dr. He Baicheng (Chongqing Key Laboratory of Biochemistry and Molecular Pharmacology, China) for providing the recombinant adenovirus of BMP9.

\section{Authors' contributions}

All authors read and approved the final manuscript.

\section{Funding}

This work was supported by the National Natural Science Foundation of China (81101360), the Shanghai Science and Technology Commission Fund (16ZR1422000 and 18411964500), the Shanghai Jiaotong University Medical and Industrial Cross Fund (YG2015MS67) and the Shanghai Sailing Program (18YF1415700).

\section{Availability of data and materials}

The datasets used and/or analysed during the current study are available from the corresponding author on reasonable request.

Ethics approval and consent to participate

This research has been approved by the Ethics Committee of Xinhua Hospital Affiliated to Shanghai Jiaotong University.

Consent for publication

Not applicable. 


\section{Competing interests}

The authors declare that they have no competing interests.

\section{Author details}

'Department of Geriatrics, Xinhua Hospital, Shanghai Jiaotong University School of Medicine, Shanghai 200092, China. ${ }^{2}$ Shanghai Institute for Pediatric Research, Shanghai Jiaotong University School of Medicine, Shanghai 200092, China

\section{Received: 24 January 2019 Revised: 17 November 2019} Accepted: 26 November 2019 Published online: 21 December 2019

\section{References}

1. He X, He J, Shi Y, Pi C, Yang Y, Sun Y, et al. Nicotinamide phosphoribosyltransferase (Nampt) may serve as the marker for osteoblast differentiation of bone marrow-derived mesenchymal stem cells. Exp Cell Res. 2017;352(1):45-52

2. Ho PJ, Yen ML, Tang BC, Chen CT, Yen $B L . \mathrm{H}_{2} \mathrm{O}_{2}$ accumulation mediates differentiation capacity alteration, but not proliferative decline, in senescent human fetal mesenchymal stem cells. Antioxid Redox Signal. 2013;18(15): 1895-905

3. Liu X, Qin J, Luo Q, Bi Y, Zhu G, Jiang W, et al. Cross-talk between EGF and BMP9 signalling pathways regulates the osteogenic differentiation of mesenchymal stem cells. J Cell Mol Med. 2013;17(9):1160-72.

4. Z Zhang H, Wang J, Deng F, Huang E, Yan Z, Wang Z, et al. Canonical Wnt signaling acts synergistically on BMP9-induced osteo/odontoblastic differentiation of stem cells of dental apical papilla (SCAPs). Biomaterials. 2015;39:145-54

5. Shi Y, Chen J, Karner CM, Long F. Hedgehog signaling activates a positive feedback mechanism involving insulin-like growth factors to induce osteoblast differentiation. Proc Natl Acad Sci U S A. 2015;112(15):4678-83.

6. Bolamperti S, Signo M, Spinello A, Moro G, Fraschini G, Guidobono F, et al. $\mathrm{GH}$ prevents adipogenic differentiation of mesenchymal stromal stem cells derived from human trabecular bone via canonical Wnt signaling. Bone. 2018;112:136-44.

7. Mi LZ, Brown CT, Gao Y, Tian Y, Le VQ, Walz T, et al. Structure of bone morphogenetic protein 9 procomplex. Proc Natl Acad Sci U S A. 2015; 112(12):3710-5.

8. Cooper GS, Kou TD. Risk of cancer after lumbar fusion surgery with recombinant human bone morphogenic protein-2 (rh-BMP-2). Spine. 2013; 38(21):1862-8.

9. Khosla S, Westendorf JJ, Oursler MJ. Building bone to reverse osteoporosis and repair fractures. J Clin Invest. 2008;118(2):421-8.

10. Morine KJ, Qiao X, York S, Natov PS, Paruchuri V, Zhang Y, et al. Bone morphogenetic protein 9 reduces cardiac fibrosis and improves cardiac function in heart failure. Circulation. 2018;138(5):513-526.

11. Ola R, Kunzel SH, Zhang F, Genet G, Chakraborty R, Pibouin-Fragner L, et al. SMAD4 prevents flow induced arterial-venous malformations by inhibiting casein kinase 2. Circulation. 2018;138(21):2379-94.

12. Liu Y, Liu Y, Zhang R, Wang X, Huang F, Yan Z, et al. All-trans retinoic acid modulates bone morphogenic protein 9-induced osteogenesis and adipogenesis of preadipocytes through BMP/Smad and Wnt/beta-catenin signaling pathways. Int J Biochemistry Cell Biol. 2014;47:47-56.

13. Yang F, Yang L, Li Y, Yan G, Feng C, Liu T, et al. Melatonin protects bone marrow mesenchymal stem cells against iron overload-induced aberrant differentiation and senescence. J Pineal Res. 2017;63(3):e12422.

14. Zhou L, Chen X, Liu T, Gong Y, Chen S, Pan G, et al. Melatonin reverses H2 $\mathrm{O} 2$-induced premature senescence in mesenchymal stem cells via the SIRT1-dependent pathway. J Pineal Res. 2015;59(2):190-205.

15. Luchetti F, Canonico B, Bartolini D, Arcangeletti M, Ciffolilli S, Murdolo G, et al. Melatonin regulates mesenchymal stem cell differentiation: a review. J Pineal Res. 2014;56(4):382-97.

16. Nakade $\mathrm{O}$, Koyama H, Ariji H, Yajima A, Kaku T. Melatonin stimulates proliferation and type I collagen synthesis in human bone cells in vitro. J Pineal Res. 1999;27(2):106-10.

17. Basoli V, Santaniello S, Cruciani S, Ginesu GC, Cossu ML, Delitala AP, et al, Melatonin and vitamin D interfere with the adipogenic fate of adiposederived stem cells. Int J Mol Sci. 2017;18(5):981.

18. Shuai $Y$, Liao L, Su X, Yu Y, Shao B, Jing H, et al. Melatonin treatment improves mesenchymal stem cells therapy by preserving stemness during long-term in vitro expansion. J Pineal Res. 2016;6(11):1899-917.
19. Ping Z, Hu X, Wang L, Shi J, Tao Y, Wu X, et al. Melatonin attenuates titanium particle-induced osteolysis via activation of Wnt/beta-catenin signaling pathway. Acta Biomater. 2017;51:513-25.

20. Park KH, Kang JW, Lee EM, Kim JS, Rhee YH, Kim M, et al. Melatonin promotes osteoblastic differentiation through the BMP/ERKWnt signaling pathways. J Pineal Res. 2011;51(2):187-94.

21. Radio NM, Doctor JS, Witt-Enderby PA. Melatonin enhances alkaline phosphatase activity in differentiating human adult mesenchymal stem cells grown in osteogenic medium via MT2 melatonin receptors and the MEK ERK (1/2) signaling cascade. J Pineal Res. 2006;40(4):332-42.

22. Tsukamoto N, Otsuka F, Ogura-Ochi K, Inagaki K, Nakamura E, Toma K, et al. Melatonin receptor activation suppresses adrenocorticotropin production via BMP-4 action by pituitary AtT20 cells. Mol Cell Endocrinol. 2013;375(1-2): $1-9$.

23. Nakamura E, Otsuka F, Terasaka T, Inagaki K, Hosoya T, Tsukamoto-Yamauchi $\mathrm{N}$, et al. Melatonin counteracts BMP-6 regulation of steroidogenesis by rat granulosa cells. J Steroid Biochem Mol Biol. 2014;143:233-9.

24. Han Y, Kim YM, Kim HS, Lee KY. Melatonin promotes osteoblast differentiation by regulating Osterix protein stability and expression. Sci Rep. 2017;7(1):5716.

25. Cho YA, Noh K, Jue SS, Lee SY, Kim EC. Melatonin promotes hepatic differentiation of human dental pulp stem cells: clinical implications for the prevention of liver fibrosis. J Pineal Res. 2015;58(1):127-35.

26. Huang E, Zhu G, Jiang W, Yang K, Gao Y, Luo Q, et al. Growth hormone synergizes with BMP9 in osteogenic differentiation by activating the JAK STAT/IGF1 pathway in murine multilineage cells. J Bone Miner Res. 2012; 27(7):1566-75.

27. Gao Y, Huang E, Zhang H, Wang J, Wu N, Chen X, et al. Crosstalk between Wnt/beta-catenin and estrogen receptor signaling synergistically promotes osteogenic differentiation of mesenchymal progenitor cells. PLoS One. 2013, 8(12):e82436.

28. Soleimani M, Nadri S. A protocol for isolation and culture of mesenchymal stem cells from mouse bone marrow. Nat Protoc. 2009;4(1):102-6.

29. Wang D, Ma W, Wang F, Dong J, Wang D, Sun B, et al. Stimulation of Wnt/ beta-catenin signaling to improve bone development by Naringin via interacting with AMPK and Akt. Cell Physiol Biochem. 2015;36(4):1563-76.

30. Lee $\mathrm{S}$, Le NH, Kang D. Melatonin alleviates oxidative stress-inhibited osteogenesis of human bone marrow-derived mesenchymal stem cells through AMPK activation. Int J Med Sci. 2018;15(10):1083-91.

31. Lamplot JD, Qin J, Nan G, Wang J, Liu X, Yin L, et al. BMP9 signaling in stem cell differentiation and osteogenesis. Am J Stem Cells. 2013;2(1):1-21.

32. Chen L, Zou X, Zhang RX, Pi CJ, Wu N, Yin LJ, et al. IGF1 potentiates BMP9induced osteogenic differentiation in mesenchymal stem cells through the enhancement of BMP/Smad signaling. BMB Rep. 2016;49(2):122-7.

33. Liu J, Zhou H, Fan W, Dong W, Fu S, He H, et al. Melatonin influences proliferation and differentiation of rat dental papilla cells in vitro and dentine formation in vivo by altering mitochondrial activity. J Pineal Res. 2013;54(2):170-8.

34. Maria S, Samsonraj RM, Munmun F, Glas J, Silvestros M, Kotlarczyk MP, et al. Biological effects of melatonin on osteoblast/osteoclast cocultures, bone, and quality of life: Implications of a role for MT2 melatonin receptors, MEK1/ 2, and MEK5 in melatonin-mediated osteoblastogenesis. J Pineal Res. 2018; 64(3):e12465.

35. Amstrup AK, Sikjaer T, Heickendorff L, Mosekilde L, Rejnmark L. Melatonin improves bone mineral density at the femoral neck in postmenopausal women with osteopenia: a randomized controlled trial. J Pineal Res. 2015; 59(2):221-9.

36. Zhang L, Su P, Xu C, Chen C, Liang A, Du K, et al. Melatonin inhibits adipogenesis and enhances osteogenesis of human mesenchymal stem cells by suppressing PPARgamma expression and enhancing Runx2 expression. J Pineal Res. 2010;49(4):364-72.

37. Zhang L, Zhang J, Ling Y, Chen C, Liang A, Peng Y, et al. Sustained release of melatonin from poly (lactic-co-glycolic acid) (PLGA) microspheres to induce osteogenesis of human mesenchymal stem cells in vitro. J Pineal Res. 2013;54(1):24-32.

38. Wang B, Wen H, Smith W, Hao D, He B, Kong L. Regulation effects of melatonin on bone marrow mesenchymal stem cell differentiation. J Cell Physiol. 2019;234(2):1008-15.

39. Liu X, Gong Y, Xiong K, Ye Y, Xiong Y, Zhuang Z, et al. Melatonin mediates protective effects on inflammatory response induced by interleukin-1 beta in human mesenchymal stem cells. J Pineal Res. 2013;55(1):14-25. 
40. He F, Liu X, Xiong K, Chen S, Zhou L, Cui W, et al. Extracellular matrix modulates the biological effects of melatonin in mesenchymal stem cells. J Endocrinol. 2014;223(2):167-80.

41. Lee WC, Guntur AR, Long F, Rosen CJ. Energy metabolism of the osteoblast: implications for osteoporosis. Endocr Rev. 2017;38(3):255-66.

42. Depner CM, Melanson EL, McHill AW, Wright KP Jr. Mistimed food intake and sleep alters 24-hour time-of-day patterns of the human plasma proteome. Proc Natl Acad Sci U S A. 2018;115(23):E5390-e9.

43. de Farias TS, de Oliveira AC, Andreotti S, do Amaral FG, Chimin P, de Proenca AR, et al. Pinealectomy interferes with the circadian clock genes expression in white adipose tissue. J Pineal Res. 2015;58(3):251-61.

44. Cipolla-Neto J, Amaral FG, Afeche SC, Tan DX, Reiter RJ. Melatonin, energy metabolism, and obesity: a review. J Pineal Res. 2014;56(4):371-81.

45. Szewczyk-Golec K, Wozniak A, Reiter RJ. Inter-relationships of the chronobiotic, melatonin, with leptin and adiponectin: implications for obesity. J Pineal Res. 2015;59(3):277-91.

46. Zhao J, Yue W, Zhu MJ, Sreejayan N, Du M. AMP-activated protein kinase (AMPK) cross-talks with canonical Wnt signaling via phosphorylation of beta-catenin at Ser 552. Biochem Biophys Res Commun. 2010;395(1):146-51

47. Chen $\mathrm{H}$, Liu X, Chen H, Cao J, Zhang L, Hu X, et al. Role of SIRT1 and AMPK in mesenchymal stem cells differentiation. Ageing Res Rev. 2014;13:55-64.

48. Shah M, Kola B, Bataveliji A, Arnett TR, Viollet B, Saxon L, et al. AMPactivated protein kinase (AMPK) activation regulates in vitro bone formation and bone mass. Bone. 2010:47(2):309-19.

49. Boufroura FZ, Le Bachelier C, Tomkiewicz-Raulet C, Schlemmer D, Benoist JF, Grondin P, et al. A new AMPK activator, GSK773, corrects fatty acid oxidation and differentiation defect in CPT2-deficient myotubes. Hum Mol Genet. 2018;27(19):3417-33.

50. Wang YG, Qu XH, Yang Y, Han XG, Wang L, Qiao H, et al. AMPK promotes osteogenesis and inhibits adipogenesis through AMPK-Gfi1-OPN axis. Cell Signal. 2016;28(9):1270-82.

51. Chava S, Chennakesavulu S, Gayatri BM, Reddy ABM. A novel phosphorylation by AMP-activated kinase regulates RUNX2 from ubiquitination in osteogenesis over adipogenesis. Cell Death Dis. 2018;9(7):754.

52. Luo Q, Kang Q, Si W, Jiang W, Park JK, Peng Y, et al. Connective tissue growth factor (CTGF) is regulated by Wnt and bone morphogenetic proteins signaling in osteoblast differentiation of mesenchymal stem cells. J Biol Chem. 2004;279(53):55958-68.

53. Si W, Kang Q, Luu HH, Park JK, Luo Q, Song WX, et al. CCN1/Cyr61 is regulated by the canonical Wnt signal and plays an important role in Wnt3A-induced osteoblast differentiation of mesenchymal stem cells. Mol Cell Biol. 2006;26(8):2955-64

54. Tang N, Song WX, Luo J, Luo X, Chen J, Sharff KA, et al. BMP-9-induced osteogenic differentiation of mesenchymal progenitors requires functional canonical Wnt/beta-catenin signalling. J Cell Mol Med. 2009;13(8b):2448-64.

55. Wang $L$, Di $L$. Wnt/beta-catenin mediates AICAR effect to increase GATA3 expression and inhibit adipogenesis. J Biol Chem. 2015;290(32):19458-68.

56. Zhao JX, Yue WF, Zhu MJ, Du M. AMP-activated protein kinase regulates beta-catenin transcription via histone deacetylase 5. J Biol Chem. 2011; 286(18):16426-34.

57. Lee YH, Kim JS, Kim JE, Lee MH, Jeon JG, Park IS, et al. Nanoparticle mediated PPARgamma gene delivery on dental implants improves osseointegration via mitochondrial biogenesis in diabetes mellitus rat model. Nanomed. 2017;13(5):1821-32.

\section{Publisher's Note}

Springer Nature remains neutral with regard to jurisdictional claims in published maps and institutional affiliations.

Ready to submit your research? Choose BMC and benefit from:

- fast, convenient online submission

- thorough peer review by experienced researchers in your field

- rapid publication on acceptance

- support for research data, including large and complex data types

- gold Open Access which fosters wider collaboration and increased citations

- maximum visibility for your research: over $100 \mathrm{M}$ website views per year

At $\mathrm{BMC}$, research is always in progress.

Learn more biomedcentral.com/submissions 